\title{
Identification of relaxation parameter of a physical model of vein from fluid transient experiment
}

\author{
David Hromádka ${ }^{1, a}$, Hynek Chlup ${ }^{1}$ and Rudolf Žitný ${ }^{2}$ \\ ${ }^{1}$ Czech Technical University in Prague, Faculty of Mechanical Engineering, Dept. of Mechanics, Biomechanics and \\ Mechatronics, Technická 4, 166 07, Prague 6, Czech Republic \\ ${ }^{2}$ Czech Technical University in Prague, Faculty of Mechanical Engineering, Dept. of Process Engineering, Technická 4, \\ 166 07, Prague 6, Czech Republic
}

\begin{abstract}
This paper presents a new fluid transient inflation experiment applied on a physical model of vein (short latex tube, $5 \mathrm{~mm}$ diameter). Aim of experiments is assessment of wall viscous behaviour from attenuated pulsation of the tested sample. Experimental data obtained from dynamic test are compared with numerical simulation and a viscoelastic parameter of Haslach constitutive model is identified. It is verified that the viscoelasticity of wall has a greater impact to the damping of pulsation than the viscosity of water filling the sample and the attached capillary. Volume of sample depends on internal pressure measured by a pressure transducer. The maximum dissipation constitutive model of viscoelastic wall sample was employed for description of viscoelastic behaviour. Frequency of natural oscillation of pressure is determined by inertia of water column within the tested sample and attached capillary and by the tested specimen stiffness. The pressure pulsations are initiated by a sudden pressure drop at water surface.
\end{abstract}

\section{Introduction}

Viscoelastic processes are non-equilibrium time dependent processes. Some energy is reversibly stored during loading and some is dissipated to heat. Several approaches exist for viscoelastic behaviour description of solids. One approach is utilization of the hereditary integral formulation based on Boltzmann superposition principle for modelling nonlinear viscoelastic behaviour developed by Coleman and Noll [1] and used for soft tissue by Fung [2] who named this approach as Quasilinear viscoelasticity (QLV). Many researchers adopted and adapted QLV theory to fit the responses of soft tissues Abramowitch and Woo [3], Funk et. al. [4], Lynch et. al. [5], Sarver et. al. [6], Toms et. al. [7], Valdez-Jasso [8]. There are also phenomenological models that are derived from parallel or serial connection of elastic springs and viscous dampers Valdez-Jasso [9], Bessems [10]. The transversely isotropic viscohyperelastic material was introduced in Limbert and Middleton work [11]. The mechanical formulation is based on a definition of a general Helmholtz free energy function, which is a sum of hyperelastic and viscous potential. Their approach is capable to describe anisotropic viscous behaviour also. Other approach is formulation viscoelastic processes in the state variables, which lead to evolution equations, and it is presented in Holzapfel work $[12,13]$ where the state variables are presented as inelastic strains or stresses. The foundations of this approach are still derived from phenomenological models of connected springs and dashpots. Haslach [14] introduced a new class nonequilibrium thermoviscoelastic evolution equation based on long-term behaviour and a maximum dissipation principle for polymers, rubbers and soft tissues. The nonlinear evolution equations (1) for thermoviscoelastic behaviour in terms of state variables, $x_{i}$, and control variables, $y_{i}$, (represented by volume $V$ and pressure $p$ for example) are generated from long-term constitutive models represented by an energy function $\Psi$ used for elasticity description, see below.

$$
\begin{aligned}
& \left(\begin{array}{c}
d x_{1} / d t \\
d x_{2} / d t \\
\vdots \\
d x_{3} / d t
\end{array}\right)= \\
& -k \cdot\left(\begin{array}{cccc}
\partial^{2} \psi / \partial x_{1}^{2} & \partial^{2} \psi /\left(\partial x_{1} \partial x_{2}\right) & \ldots & \partial^{2} \psi /\left(\partial x_{1} \partial x_{n}\right) \\
\partial^{2} \psi /\left(\partial x_{2} \partial x_{1}\right) & \partial^{2} \psi / \partial x_{2}^{2} & \ldots & \partial^{2} \psi /\left(\partial x_{2} \partial x_{n}\right) \\
\ldots & \ldots & \ldots & \ldots \\
\partial^{2} \psi /\left(\partial x_{n} \partial x_{1}\right) & \partial^{2} \psi /\left(\partial x_{n} \partial x_{2}\right) & \cdots & \partial^{2} \psi / \partial x_{n}^{2}
\end{array}\right) \\
& \left(\begin{array}{c}
-y_{1}(t)+\partial \psi / \partial x_{1} \\
-y_{2}(t)+\partial \psi / \partial x_{2} \\
\ldots \\
-y_{n}(t)+\partial \psi / \partial x_{n}
\end{array}\right)
\end{aligned}
$$

\footnotetext{
${ }^{\mathrm{a}}$ Corresponding author: david.hromadka@fs.cvut.cz
} 
The different dynamic experimental measurement of tubular specimen inelastic response is presented in Valdes-Jasso [8] and in Bessems [9].

This paper deals with a fluid transient experiment on the physical model of vein. Mathematical model formulation is simplified by the fact that only a small part of system boundary is flexible (tested section of elastic tube is very short comparing with a long rigid piping in experimental setup). Thus the effect of a moving pulse wave can be neglected and the whole system can be approximated by the 'windkessel' (0-D) model that is by a system of ordinary differential equations. Only one specimen of elastic tube was tested: a simple latex tube with small inner diameter. The objective is to obtain a relaxation parameter of the Haslach's constitutive model for description of the tested specimen during a transient loading and to assess whether fluid friction or tube wall viscoelasticity has a greater influence on attenuation of pressure pulsation.

\section{Methods}

\subsection{Measurement of elastic response}

Inflation test was carried out to obtain dependence between pressure and volume, $p_{l e}(V)$. The tested sample is connected to the capillary filled with water (water column height is approximately $h_{\text {inf }} \approx 0.2 \mathrm{~m}$ ) and the pressure vessel. Pressure increase recorded with pressure transducer is accompanied by volume increase within specimen measured as change of water column $h_{\text {inf }}$ in capillary. Measurement setup is schematically showed on figure 1

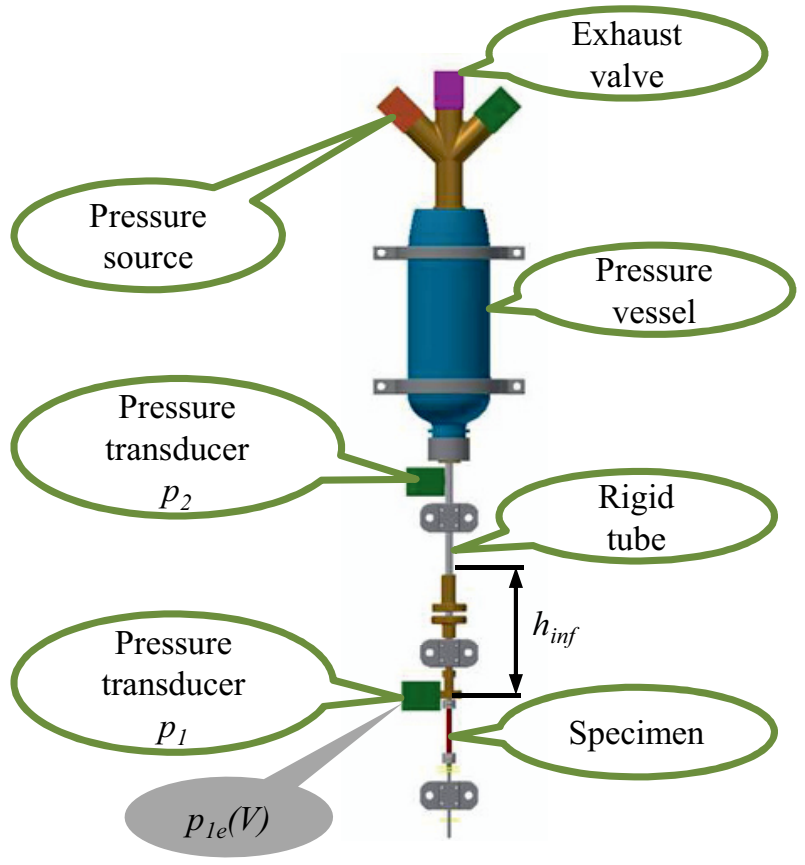

Figure 1. Experimental setup scheme used for the inflation test and the dynamic test

\subsection{Measurement of dynamic response}

The pressure vessel, connected rigid tube and the compliant sample is pressurised to demanded pressure level $p_{2}(0)$ at the beginning of experiment. Instantaneous opening and closing electromagnetic valve on the outlet of pressure tank causes pressure drop and initiate the dynamic experiment. The response to the pressure drop is expulsion of water volume from specimen and it leads to a movement of the water column. Gravity, inertia of excited water column and specimen compliance cause the oscillation of water column accompanied by pressure pulsation. The history of pressure upon the water level and pressure pulsations is recorded with pressure sensors $p_{2}, p_{1}$. The same experimental setup was used (see figure 1) but in this case with greater value of water column height $h_{p}$ (approximately $1 \mathrm{~m}$ ).

\subsection{Mathematical model}

Mathematical description of experiment is split into two parts the description of viscoelastic behaviour of the specimen and the description of flow dynamics.

\subsubsection{Viscoelastic model of specimen}

Viscoelastic model is derived from principle of maximum dissipation in accordance to Haslach [14]. The key to formulation of viscoelastic model is construction of generalized energy function (2)

$$
\psi^{*}\left(V ; p_{1}\right)=\psi(V)-V p_{1}=\int_{V_{0}}^{V} p_{1 e} d V-V p_{1}
$$

and the definition of affinity (3) (thermodynamic force) which drives non-equilibrium process and provides the information how far is non-equilibrium state from the equilibrium.

$$
X=\partial \psi^{*} / \partial V=\partial \psi / \partial V-p_{1}
$$

The affinity $X$ is equal to zero only in case of equilibrium state $\left(p_{1 e}=p_{1}\right)$. The function $\psi(V)$ corresponds to the deformation energy. The flux (4) is defined as affinity time derivative and describes the response of tubular viscoelastic thermostatic system

$$
\dot{X}=-k_{V} \cdot \partial \psi^{*} / \partial X
$$

with using chain rule applied on (4) we obtain equation (5).

$$
\partial X / \partial V \cdot \dot{V}=-k_{V} \cdot \partial \psi^{*} / \partial V \cdot \partial V / \partial X
$$

The equation (5) is rewritten into the form (6) after substitution first spatial derivative of generalized energy function with equation (3) and awareness of the fact that spatial derivative of affinity with respect to the volume $V$ is the derivative of second order $\partial^{2} \psi / \partial V^{2}$.

$$
p_{1}(V, \dot{V})=\partial \psi / \partial V+1 / k_{V} \cdot\left(\partial^{2} \psi / \partial V^{2}\right)^{2} \cdot \dot{V}
$$


The relaxation coefficient $k_{V}$ must be evaluated from the observed attenuation of oscillations. Limit cases of relaxation parameter amplitude ( $k_{V}$ is sufficiently close to 0 or $k_{V}$ approaches infinity) correspond to elastic body or viscous tube. The equation (6) is evolution equation that describes viscoelastic behaviour of viscoelastic tubular specimen and represents material with ideal memory (type of Kelvin-Voight material).

First spatial derivative is specimen stiffness characteristic $G_{e l}$ on the right side (6) obtained from inflation test.

$$
G_{e l}=\partial \psi / \partial V=p_{1}(V)
$$

The function accountable for viscous response of specimen $G_{v}$ is a quadrature of first spatial derivation of (7) with respect to $V$.

$$
G_{v}=\left(\partial G_{e l} / \partial V\right)^{2}=\left(G_{e l}^{\prime}\right)^{2}
$$

The evolution equation (6) can be written in more compact form now

$$
p_{1}(V, \dot{V})=G_{e l}+1 / k_{V} \cdot\left(G_{e l}^{\prime}\right)^{2} \cdot \dot{V}
$$

Haslach assumes that equilibrium state and nonequilibrium state lies on the same state surface described by generalized energy function (2) obtained from perfect knowledge of equilibrium state between state variables (in this case between pressure and volume at steady state). The evolution of state variable at time from nonequilibrium state towards equilibrium state is selected in such way that the entropy production is maximal. The elastic part $G_{e l}$ can be described by a hyper-elastic model (assuming symmetrically deformed circular pipe) or by using an experimentally determined function from the quasi-static inflation test as relation between pressure and volume (7).

\subsubsection{Fluid dynamics}

The radial velocity profile during transient flow within capillary is no longer simple parabolic profile in the case of high Womersley number $(10)(W o \approx 4)$

$$
W_{o}=R_{p} \cdot(\rho \omega / \mu)^{0.5}
$$

The difference between flow-rate calculated from parabolic velocity profile and polynomial velocity profile of fourth order (11) is approximately $20 \%$ for this pulsatile flow case.

$$
w_{p}(r, t)=c_{1}(t) \cdot\left(1-r^{2} / R_{p}^{2}\right)+c_{2}(t) \cdot\left(r^{2} / R_{p}^{2}-r^{4} / R_{p}^{4}\right)
$$

The polynomial function (11) is capable to describe flow reversal at the wall region, which is a situation typical for quickly changing axial gradient of pressure within the capillary with inner radius $R_{p}$. The first term on the right side is a parabolic function (steady state velocity profile) and the second term is a wall function (describes a possible reversal flow at the wall).

The solution for pulsatile flow is based on highly simplified form of the Navier-Stokes equations (12). The assumptions that lead to (12) follows from concept of long straight tube with circular cross section and absence of any external forces that would cause flow rotation.

$$
\begin{aligned}
& \rho \cdot \partial w_{p}(r, t) / \partial t= \\
& =-\partial p / \partial z-\rho g+\mu / r \cdot \partial / \partial r\left(r \cdot \partial w_{p}(r, t) / \partial r\right)
\end{aligned}
$$

Navier-Stokes equation (12) is applied on (11) and partial differential equation (13) for unknown amplitudes $c_{l}(t)$, $c_{2}(t)$ of basis functions is obtained

$$
\begin{aligned}
& \rho \cdot \partial c_{1}(t) / \partial t \cdot\left(1-r^{2} / R_{p}^{2}\right)+ \\
& +\rho \cdot \partial c_{2}(t) / \partial t \cdot\left(r^{2} / R_{p}^{2}-r^{4} / R_{p}^{4}\right)= \\
& =-\partial p / \partial z-\rho g+4 \mu / R_{p}^{2} \cdot\left(c_{2}(t)-c_{1}(t)\right)- \\
& -16 \mu r^{2} / R_{p}^{4} \cdot c_{2}(t)
\end{aligned}
$$

The differential equation (13) is solved by method of weighted residual

$$
\begin{aligned}
& \int_{0}^{R_{p}} w_{1} \cdot r e s \cdot d r=0 \\
& \int_{0}^{R_{p}} w_{2} \cdot r e s \cdot d r=0
\end{aligned}
$$

where residual denoted res correspond to equation bellow

$$
\begin{aligned}
& r e s=\rho \cdot \partial c_{1}(t) / \partial t \cdot\left(1-r^{2} / R_{p}^{2}\right)+ \\
& +\rho \cdot \partial c_{2}(t) / \partial t \cdot\left(r^{2} / R_{p}^{2}-r^{4} / R_{p}^{4}\right)+\partial p / \partial z+ \\
& +\rho g-4 \mu / R_{p}^{2} \cdot\left(c_{2}(t)-c_{1}(t)\right)+16 \mu r^{2} / R_{p}^{4} \cdot c_{2}(t)
\end{aligned}
$$

Weighted functions $w_{1}, w_{2}$ were derived from Galerkin method

$$
\begin{aligned}
& w_{1}=d w_{p} / d c_{1} \\
& w_{2}=d w_{p} / d c_{2}
\end{aligned}
$$

The solution of (14) leads to the system of ordinary differential equations in the form

$$
\begin{aligned}
& 8 / 15 \rho \cdot d c_{1}(t) / d t+8 / 105 \rho \cdot d c_{2}(t) / d t+2 / 3(\partial p / \partial z+\rho g)+ \\
& +8 / 3 \mu / R_{p}^{2} \cdot c_{1}(t)-8 / 15 \mu / R_{p}^{2} \cdot c_{2}(t)=0 \\
& 8 / 105 \rho \cdot d c_{1}(t) / d t+8 / 315 \rho \cdot d c_{2}(t) / d t+2 / 15(\partial p / \partial z+\rho g)+ \\
& +8 / 15 \mu / R_{p}^{2} \cdot c_{1}(t)+8 / 21 \mu / R_{p}^{2} \cdot c_{2}(t)=0
\end{aligned}
$$


It is more convenient to work with flowrate therefore it is necessary expressed velocities $c_{1}(t), c_{2}(t)$ in terms of flowrate. Flowrates (19) are given by integration of (18)

$$
\begin{aligned}
& q_{p}=\int_{0}^{2 \pi} d \varphi \int_{0}^{R_{p}} r w_{p} d r= \\
& =\pi c_{1}(t) R_{p}^{2} / 2+\pi c_{2}(t) R_{p}^{2} / 6 \\
& q_{w}=\int_{0}^{2 \pi} d \varphi \int_{0}^{R_{p}} r c_{2}(t) \cdot\left(r^{2} / R_{p}^{2}-r^{4} / R_{p}^{4}\right) d r= \\
& =\pi c_{2}(t) R_{p}^{2} / 6
\end{aligned}
$$

The functions $c_{l}(t), c_{2}(t)$ can be expressed in terms of flowrates $q_{p}$ and $q_{w}$ after small manipulation.

$$
\left(\begin{array}{l}
c_{1}(t) \\
c_{2}(t)
\end{array}\right)=2 / \pi R_{p}^{2}\left(\begin{array}{cc}
1 & -1 \\
0 & 3
\end{array}\right)\left(\begin{array}{l}
q_{p}(t) \\
q_{w}(t)
\end{array}\right)
$$

The final form of Navier-Stokes equations is obtained when equations (20) and (18) are combined together. The pressure slope (responsible for change of velocity profile shape) in (18) is substituted by given equation of pressure gradient $P(t)$

$$
P(t)=\left(p_{2}(t)-p_{1}(V, \dot{V})\right) / h_{p}(t)+\rho g
$$

The final form of Navier-Stokes equations is obtained when equations (20) and (18) are combined together. The pressure slope (responsible for change of velocity profile shape) in (18) is substituted by given equation of pressure gradient $P(t)$ thereafter Bernoulli's equations in terms of flowrate are obtained

$$
\begin{aligned}
& \rho R_{p}^{2} / \mu\left(\begin{array}{l}
\dot{q}_{p}(t) \\
\dot{q}_{w}(t)
\end{array}\right)= \\
& =-\left(\begin{array}{c}
7 / 8 \\
7 / 16
\end{array}\right) P(t) \pi R_{p}^{4} / \mu-\left(\begin{array}{cc}
7 & 8 \\
7 / 2 & 28
\end{array}\right)\left(\begin{array}{l}
q_{p}(t) \\
q_{w}(t)
\end{array}\right)
\end{aligned}
$$

Remark: The flowrate $q_{w}$ is only a fraction of the overall flowrate $q_{p}$ and is not directly involved in the flow balancing (it only serves to improve the accuracy). The system of ODE's (22) has the analytical solution in the case of constant pressure gradient. Only two term in (22) is unknown $\left(p_{2}(t)\right)$. The last unknown (surface pressure) $p_{2}(t)$ is measured by a pressure transducer during dynamical experiment and is fitted by a cubic natural spline (the pressure $p_{2}(t)$ is an input to the mathematical model)

$$
p_{2}(t)=f(t)
$$

The pulsatile flow described by (22) computes the flow only in region of water column $h_{p}(t)$ see figure 2 .

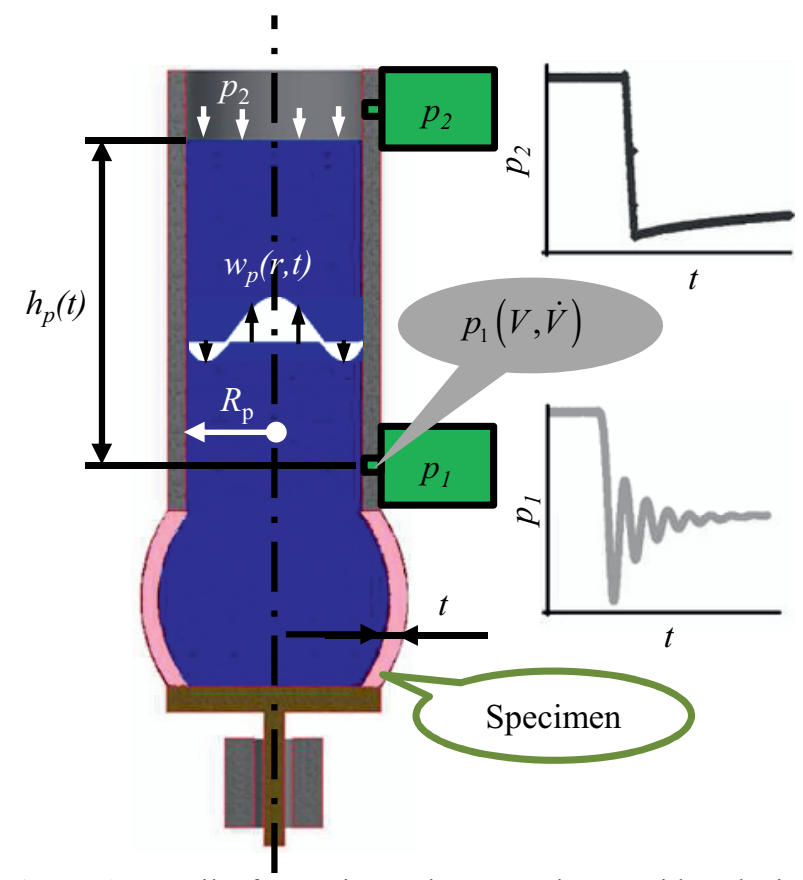

Figure 2. Detail of experimental setup scheme with velocity profile approximation and measured pressure time diagram. The rigid tube inner radius is $R_{p}=2.16 \cdot 10^{-3} \mathrm{~m}$, dimensions of specimen are inner radius $R_{i}=2.29 \cdot 10^{-3} \mathrm{~m}$, sample length $\mathrm{L}=$ $4.744 \cdot 10^{-2} \mathrm{~m}$, and wall thickness $\mathrm{t}=2.84 \cdot 10^{-4} \mathrm{~m}$.

Change of volume in the tested specimen $V$ is given by flowrate

$$
\dot{V}=-q_{p}(t)
$$

and the corresponding water column is defined as

$$
\dot{h}_{p}=q_{p}(t) / \pi R_{p}^{2}
$$

Fully implicit Euler method was used for the numerical integration of differential equations and implemented as a simple Fortran program.

Initial condition are obvious and correspond to zeros flowrates, initial height of water column $h_{p}(0)$ that is subtracted from initial pressures $p_{I}(0)$ and $p_{2}(0)$ and initial volume in tested pipe $V(0)$ evaluated from $p_{l}(V)$ characteristic.

\subsection{Frequency analysis}

Frequency analysis was carried out on time interval 1.03.0 second. The main frequency was determined as the number of peaks per time interval.

\section{Results}

\subsection{Elastic response}

The inflation test of the vein physical model revealed nonlinear pressure-volume relationship, see figure 3 . 


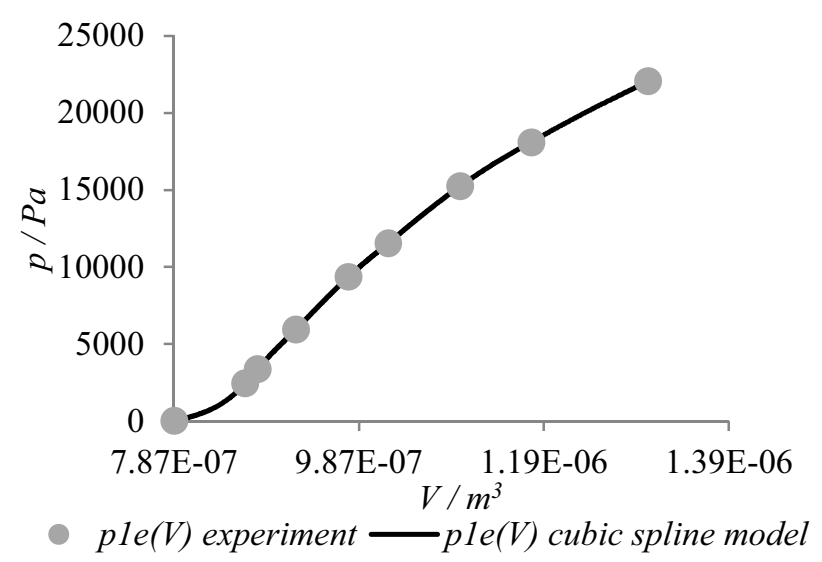

Figure 3. Inflation test results

The model (natural cubic spline model) was adopted for description of the pure elastic behaviour.

\subsection{Dynamic response}

Results from experiment and simulation are showed on figure 4 , figure 5 .

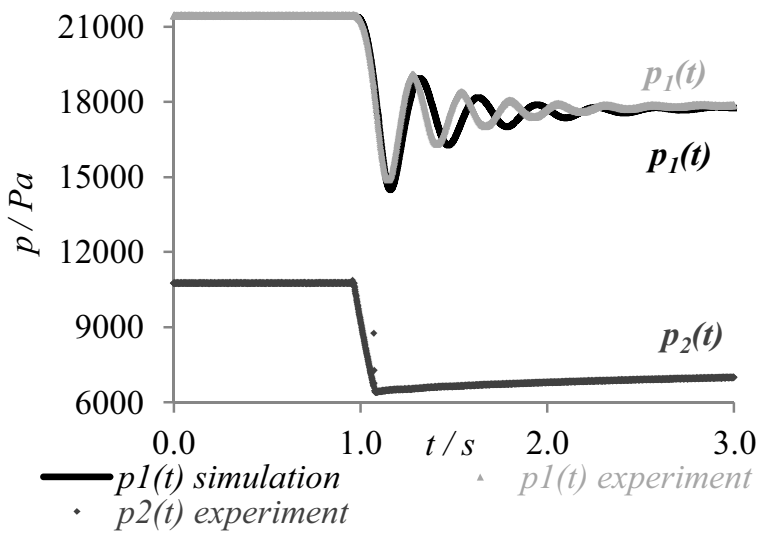

Figure 4. Measured data from dynamic test and mathematical simulation results (viscoelastic material-relaxation parameter $k_{V}$ $=5 \cdot 10^{12} \mathrm{~Pa} \mathrm{~m}^{-3} \mathrm{~s}^{-1}$, water viscosity $\mu=0.001 \mathrm{~Pa} \mathrm{~s}$, frequency of experiment $f=3.87 \mathrm{~Hz}$, frequency of simulation $f=3.19 \mathrm{~Hz}$ ).

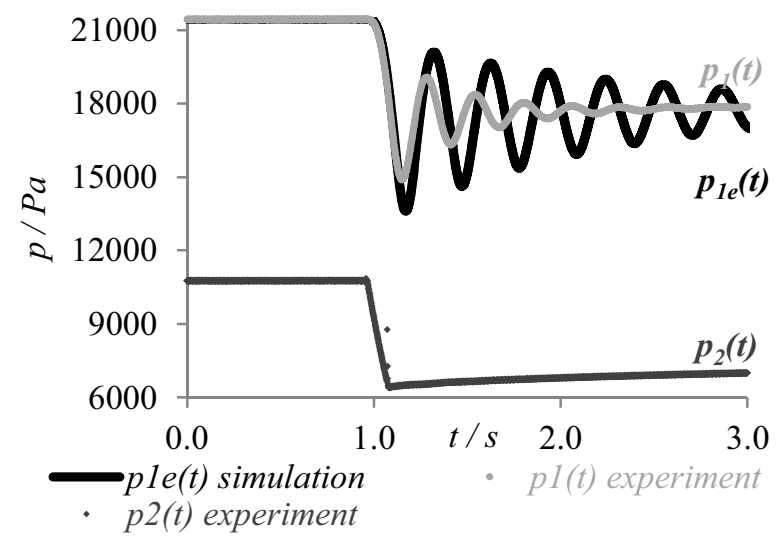

Figure 5. The same data from experiment as on figure 4 with different simulation results (elastic material-relaxation parameter $k_{V}=3 \cdot 10^{30} \mathrm{~Pa} \mathrm{~m}^{-3} \mathrm{~s}^{-1}$, water viscosity $\mu=0.001 \mathrm{~Pa}$ $\mathrm{s}$, frequency of experiment $f=3.87 \mathrm{~Hz}$, frequency of simulation $f=3.24 \mathrm{~Hz})$
Points denoted $p_{l}(t), p_{2}(t)$ represent recorded pressures during the fluid transient experiment. Two numerical simulations with different value of manually estimated relaxation parameter were performed the first one with amplitude $k_{V}=5 \cdot 10^{12} \mathrm{~Pa} \mathrm{~m}^{-3} \mathrm{~s}^{-1}$ (figure 4) and second with value $k_{V}=3 \cdot 10^{30} \mathrm{~Pa} \mathrm{~m} 3 \mathrm{~s}^{-1}$ (figure 5). The simulation carried out with higher relaxation coefficient serves to suppression of specimen viscous behaviour. Measured natural frequency was $f=3.87 \mathrm{~Hz}$. The simulation based on the natural cubic spline model gave the frequency $f=3.19 \mathrm{~Hz}$ for viscoelastic material and frequency obtained from simulation with purely elastic material was $f=3.24 \mathrm{~Hz}$.

\section{Conclusion}

Inflation test data was fitted successfully. Comparison of recorded pressure pulsations with simulations enables us to identify the value of relaxation parameter of the attenuation function $G_{v}$. The best fit corresponds to simulation with relaxation coefficient $k_{V}=5 \cdot 10^{12} \mathrm{~Pa} \mathrm{~m}^{-}$ $3 \mathrm{~s}^{-1}$. The simulation with $k_{V}=30 \cdot 10^{30} \mathrm{~Pa} \mathrm{~m}^{-3} \mathrm{~s}^{-1}$ shows that the attenuation caused by wall viscoelasticity plays important role. We showed that the experimental setup presented in this study is suitable for measurement of inelastic responses. The discrepancy (approx. 18\%) in measured natural frequency and simulated can be caused by the fact that flow within region of specimen, capillary forces and inertia of the wall are neglected or used viscoelastic model isn't suitable for description of this type test.

\section{Acknowledgment}

This work has been supported by grant SGS SGS10/247/OHK2/3T/12 and project Ministry of Health CR NT133024.

\section{Symbols}

$\mu$ dynamic viscosity, Pas;

$\rho$ water density, $\mathrm{kg} \mathrm{m}^{-3}$;

$\omega$ angular frequency, $s^{-1}$;

$\psi$ deformation energy, $J$;

$\psi^{*}$ generalized energy function, $J$;

$G_{e l}$ stiffness characteristic (elastic function of specimen), $\mathrm{Pa}$;

$G_{e l}^{\prime}$ first spatial derivative of stiffness characteristic, $\mathrm{Pam}^{-3}$;

$G_{v}$ viscous function, $\mathrm{Pa}^{2} \mathrm{~m}^{-6}$;

$c_{1}$ amplitude of parabolic velocity basis function ,

$m s^{-1}$;

$c_{2}$ amplitude of velocity basis function near the wall, $m s^{-1}$;

$g$ gravity acceleration, $m s^{-2}$; 
$h_{\text {inf }}$ water column used for inflation test, $m$;

$h_{p}$ water column used for dynamic test, $m$;

$\dot{h}_{p}$ evolution of water column, $\mathrm{ms}^{-1}$;

$k$ relaxation coefficient, unit is derived from form of generalized function

$k_{V}$ relaxation coefficient, $\mathrm{Pam}^{-3} s^{-1}$;

$p$ pressure, $\mathrm{Pa}$;

$p_{1 e}(V)$ specimen stiffness characteristic $\quad, \mathrm{Pa}$;

$p_{1}(V, \dot{V})$ viscoelastic constitutive model, $\mathrm{Pa}$;

$p_{1}(t)$ pressure oscillations, $\mathrm{Pa}$;

$p_{2}(t)$ surface pressure on the water level, $\mathrm{Pa}$;

$P(t)$ pressure gradient, $\mathrm{kg} \mathrm{m}^{-2} \mathrm{~s}^{-2}$;

$q_{p}$ flowrate within the rigid tube, $m^{3} s^{-1}$;

$q_{w}$ back flow within the rigid tube, $m^{3} s^{-1}$;

$\dot{q}_{p}$ evolution of flowrate within rigid tube, $m^{3} s^{-2}$;

$\dot{q}_{w}$ evolution of back flow within rigid tube, $m^{3} s^{-2}$;

$r$ coordinate of radius $R_{p}, m$;

$R_{p}$ radius of rigid pipe, $m$;

res residuum

$t$ time, $s$;

$V_{0}$ the internal volume of the sample at zero pressure, $\mathrm{m}^{3}$;

$V$ internal volume of specimen at time $\mathrm{t}, \mathrm{m}^{3}$;

$\dot{V}$ flowrate, $m^{3} s^{-1}$;

$w_{1}$ weighted function

$w_{2}$ weighted function

$w_{p}$ velocity profile approximation, $\mathrm{ms}^{-1}$;

$W_{O}$ womersley number

$X$ affinity, $\mathrm{Pa}$;

$\dot{X}$ flux, $\mathrm{Pas}^{-1}$;

$x_{i}$ conjugated variable (state variable)

$y_{i}$ conjugated variable (control variable)

$z$ axial coordinate, $m$;

\section{References}

1. B.D. Coleman, W. Noll, Rev. Mod. Phys., 33(2), (1961), pp. 239-249 .

2. Y.C. Fung, Biomechanics: Mechanical Properties of Living Tissues. Springer Berlin: Springer, (1981).

3. S.D. Abramowitch, S. L. Woo, J. biomech. Eng, Vol. 126, (2004), pp. 92-97.

4. J. R. Funk, G.W. Hall, J. Biomech. Eng., 122, (2000), pp. 15-22.

5. H.A. Lynch, W. Johannessen, J. Biomech. Eng., 125, (2003), pp. 726-31.

6. J.J. Sarver,P.S. Robinson, J. Biomech. Eng., 125, (2003), pp. 754-8.

7. S.R. Toms,G.J. Dakin, J. Biomech., 35, (2002), pp. 1411-5.
8. D. Valdez-Jasso ,. IEEE TRANSACTIONS ON BIOMEDICAL ENGINEERING, NO. 2, (2009), pp. 210-219.

9. D. Valdez-Jasso, Annals of Biomedical Engineering, No. 5., (2011), pp. 1438-1456.

10. D. Bessems, J. of Biomech., 41, (2008), pp. 284291.

11. G. Limbert, International Journal of Solids and Structures, 41, (2004), pp. 4237-4260.

12. G.A Holzapfel, T. C. Gasser, Comput. Methods Appl. Mech. Engrg., 190, (2001), pp. 4379-4403.

13. G.A. Holzapfel, I. Solids Structures, No. (20-22), (1996), pp. 3019-3034.

14. Haslach H.W. Jr.: Maximum Dissipation NonEquilibrium Thermodynamics and its Geometric Structure. Springer New York: Springer, (2011) 